\title{
Skin Temperature of Slaughter Pigs With Tail Lesions
}

\author{
Dayane Lemos Teixeira ${ }^{1,2 *}$, Laura Ann Boyle ${ }^{3}$ and Daniel Enríquez-Hidalgo ${ }^{2,4}$ \\ ${ }^{1}$ Instituto de Ciencias Agroalimentarias, Animales y Ambientales (ICA3), Universidad de O'Higgins, San Fernando, Chile, \\ ${ }^{2}$ Departamento de Ciencias Animales, Pontificia Universidad Católica de Chile, Santiago, Chile, ${ }^{3}$ Teagasc Animal and \\ Grassland Research and Innovation Centre, Fermoy, Ireland, ${ }^{4}$ Bristol Veterinary School, University of Bristol, Langford, \\ United Kingdom
}

OPEN ACCESS

Edited by:

Birte L. Nielsen,

Institut National de Recherche pour l'agriculture, l'alimentation et l'environnement (INRAE), France

Reviewed by:

Mona Lilian Vestbjerg Larsen

KU Leuven, Belgium

Mette S. Herskin,

Aarhus University, Denmark

*Correspondence:

Dayane Lemos Teixeira dadaylt@hotmail.com

Specialty section:

This article was submitted to Animal Behavior and Welfare, a section of the journal

Frontiers in Veterinary Science

Received: 30 January 2020

Accepted: 25 March 2020

Published: 30 April 2020

Citation:

Teixeira $D L$, Boyle $L A$ and Enríquez-Hidalgo D (2020) Skin Temperature of Slaughter Pigs With Tail Lesions. Front. Vet. Sci. 7:198. doi: 10.3389/fvets.2020.00198
The aim of this study was to assess the effect of tail lesion severity on skin temperature of slaughter pigs measured at the base of the tail and the ear by infrared thermography camera and to evaluate the association between the temperature measurements. Pigs were randomly selected in the lairage, containing $\sim 200$ pigs/pen. Tail lesions were scored according to severity, using a 0-4 scale. Tail lesion scores 0 and 1 were summed as it was difficult to distinguish healed lesions from mild lesions due to animal dirtiness. In total, 269 study pigs were imaged at the two locations. The effect of tail lesion score and sex of the pig on the highest temperature of the infrared image areas were analyzed using linear mixed models. Association between the tail base and ear base temperatures was evaluated using Pearson correlation. Skin temperature measured at the base of the tail was significantly lower for tails scored $0-1$ than for all other tail lesion scores $(P<$ 0.05). Pigs with tail lesion scored 2 had significantly lower skin temperatures at the base of the tail than pigs with tail lesion scored 3 or $4(P<0.05)$ while there was no difference in skin temperature at the base of the tail between pigs with tail lesion scored 3 and 4 $(P>0.05)$. Skin temperature measured at the ear base was significantly lower for pigs with tail lesion scored $0-1$ than pigs of all other tail lesion scores $(P<0.05)$ with no difference between the other scores $(P>0.05)$. Furthermore, there was an association between the two measurements $(r=0.50 ; P<0.001)$. The findings suggest that even pigs with moderate tail lesions (score 2) may have general inflammation and infection, evidenced by the elevated systemic temperature compared to pigs with none or mild tail lesion (score 0-1).

Keywords: skin temperature, tail lesions, pigs, tail biting, casualty

\section{INTRODUCTION}

Tail biting is a widespread damaging behavior performed by pigs which causes major welfare problems in intensive pig production systems $(1,2)$. As an endemic, multifactorial phenomenon (3) it is difficult to understand the specific on-farm factors that are responsible for the behavior (4). Tail biting is linked to impaired welfare in the biter because it reflects disharmony between the pig and its environment $(5)$. Being a victim is stressful $(6,7)$ and painful $(7,8)$ and in extreme cases leads to death (1). Tail bitten pigs are reluctant to spend time feeding to avoid exposing the tail (9), and thus reduce feed intake and average daily gain, resulting in lower carcass weight $(1,7,10-12)$.

Extensive investigations into the epidemiology and consequences of tail biting $(11,13,14)$ show that it also reflects deficiencies in the pigs' health status (15). Tail lesions are associated with 
inflammation and changes in stress physiology (7), and provide a route for infection $(8,9,16,17)$ that can be hematogenously disseminated to different organs $(8,9,18)$. Tail lesions present three separate routes for dissemination of infection around the body with local, in the form of pelvic abscessation or osteomyelitis, or systemic consequences: pyemia or multiple abscessation of distant anatomical locations $(9,17)$.

Tail lesions are associated with certain pathological lesions $(3,9,19-22)$, particularly with carcass abscessation $(12,18,20$, $21,23)$, but also with viscera and carcass condemnation $(22,24)$. Furthermore, welfare-related lesions, such as tail damage $(9,25)$, are associated with chronic stress (e.g., hypocortisolism) (7), which suppresses the immune system. Therefore, aside from providing an entry point for pathology, tail lesions can also contribute to an increased prevalence and duration of disease $(26,27)$, such as gastric lesions (28), and respiratory organ inflammation and disease (6).

The body surface temperature is determined by the heat exchange between the skin and environment, the metabolic activity and the blood circulation in the anatomical structure close to the body surface (29). The normal body temperature in resting pigs varies between 37.0 and $39.6^{\circ} \mathrm{C}$ (30), but under stressful situations it can increase up to $41.0^{\circ} \mathrm{C}$ (31). Previous studies have attempted to measure the skin temperature of pigs as an indicative measure of their body temperature $(32,33)$, especially using the ear base as a reference (34).

Infrared thermography cameras provide fairly accurate temperature measurements with good spatial and thermal resolution being very convenient in veterinary medicine. Infrared thermography is in use in different studies aiming to improve detection of unhealthy animals, including early identification of clinical symptoms $(34,35)$ and fever $(36)$ or high temperature $\left(>39.5^{\circ} \mathrm{C}\right)$ detection (29). Infrared thermography has the advantage over other temperature measurement methods used in veterinary medicine as it is not invasive and avoids spreading infection among sick animals. Furthermore, infrared thermography images display the distribution of surface temperature, allowing the analysis of (hot or cold) spots in selected areas for subsequent detection of possible anomalies (34). Infrared thermography images are suitable for measuring skin temperature in pigs, as the density of their hair offers several bare skinned surface areas, especially at the ear base and the udder (34).

The aim of this study was to assess the effect of tail lesion severity on skin temperature of slaughter pigs measured at the base of the tail and the ear by infrared thermography camera, and to evaluate the association between the temperature measurements. Due to systemic inflammation and/or infection, we hypothesized that increasing severity of tail lesion would be associated with increased skin temperature in the regions.

\section{MATERIALS AND METHODS}

\section{Data Collection}

The study was conducted in four experimental days during April and May 2019, with similar weather conditions, in a Chilean abattoir. Data were collected where the pigs (Large White $\mathrm{x}$
Landrace; $100-110 \mathrm{Kg}$ of body weight) were lairaged in pens at the abattoir before slaughtering (water was available but no food). Pigs were mixed prior to transport but not after arrival at the abattoir. All data were collected between 10 am and 3 pm. Data were collected from 269 study pigs. A total of $55 \%$ of the study pigs were castrated males. All pigs were tail docked $(3-5 \mathrm{~cm}$ of length). Pigs were randomly selected in the lairage, containing $\sim 200$ pigs/lairage pen. The origin of pigs (farm/batch), number of lairage pens and the period that they remained in the lairage were not recorded.

Throughout the study, the same person scored tail lesions and subsequently took a digital thermography image (DTI) and a digital image was real time recorded parallel to each thermal image through a built-in digital camera (TESTO 875-2i; Entech Industrial Solution Co., Ltd.; Thailand). The area of the tail region selected was the loin area adjacent to the base of the tail (Figures 1a,b). The ear base area was selected to be imaged (Figures 1c,d) as it is considered one of the best skin locations due to its high correlation with pig's rectal temperature (34). The tail lesion score, the sex of the pig (female or castrated male) and the number (code) of the images were registered by a second person, who also marked the pigs on the lumbar area using animal marking sticks (Raidex $\left.{ }^{(}\right)$) to track animals already measured for the study. Both observers entered into the pen by its gate and moved smoothly through the pigs.

Tail lesions were scored according to severity. The 0-4 scale (Figure 2) was adapted from Kritas and Morrison (20) and Harley et al. (37). As pigs were dirty and laired in pens without sprinkling devices, it was difficult to accurately distinguish between tail lesion scores 0 and 1; therefore, they were considered together. A minimum sample size of 50 pigs within each tail lesion score was selected. Data were collected

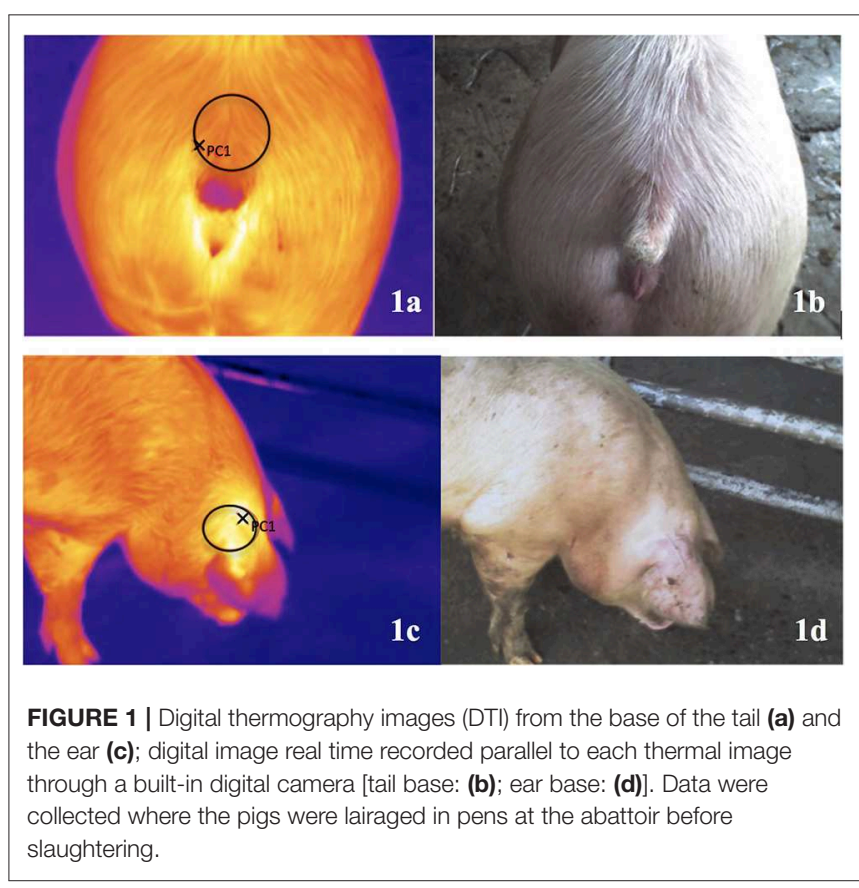



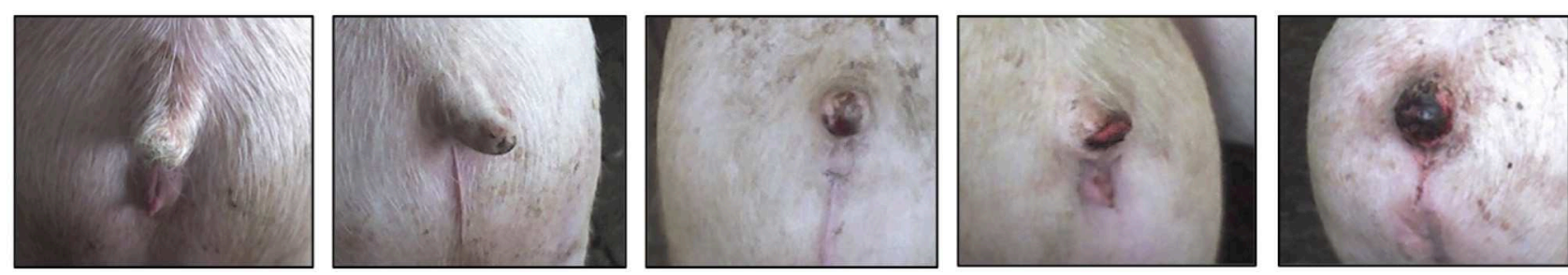

FIGURE 2 | Tail lesion scoring system adapted from Kritas and Morrison (20) and Harley et al. (37). (Scores 0-4, left to right). (0) No evidence of tail biting-clean, white unblemished tail; (1) Superficial disruption of the skin of the tail tip and/or light to darker brown marks along the length of the tail but no obvious evidence of teeth marks/puncture wounds on the tail; no swelling, no necrotic tissue; (2) Evidence of teeth marks (puncture wounds) on the tail tip and/or length; characterized by disrupted epidermis and redness but no evidence of swelling; (3) Evidence of teeth marks/puncture wounds (chewing); disrupted epidermis with redness and swelling but no necrotic tissue; (4) Open wound on the tail or where tail used to be; redness, swelling and necrotic tissue evidenced by yellowish (pus) slough or eschar (dry, black, hard necrotic tissue).

from 101 pigs with tail lesions scored as 0-1, 51 pigs with tails scored as 2, 51 pigs with tails scored 3, and 66 pigs with tails scored 4 . In all experimental days, pigs with the five different tail lesion scores were scored and imaged. All study pigs were imaged at the two locations (at the base of the tail and the ear).

\section{Capture and Analysis of Digital Thermography Images}

The thermography camera was calibrated with Iron colors, ranging from $24^{\circ} \mathrm{C}$ and $37^{\circ} \mathrm{C}$ such that those areas on the skin surface of pigs with a temperature higher than the limit temperature appeared as yellow on the images. Accuracy of the infrared thermography camera was $\pm 2{ }^{\circ} \mathrm{C}$. Emissivity (description of materials ability to emit energy by radiation) was set at 0.98 for all DTI as recommended by Soerensen et al. (38). The ambient temperature and humidity parameters were automatically recorded by the camera, together with the measured surface temperature. As all animals were lairaged in similar conditions, ambient temperature was not included in any correction formula. The distance between the equipment and the pig was targeted at $\sim 1 \mathrm{~m}$ for all measurements, which was considered to be a safe distance for both animals and observers. All animals were imaged from ground level.

All images were downloaded and analyzed using the PC Software IRSoft for TESTO thermal images (v4.5). For each image, a circle area of $\sim 10 \mathrm{~cm}$ of diameter was selected to highlight the hotspot (i.e., the highest temperature) in the selected area (Figure 1). The hotspot of the selected area for each DTI (base of the tail and the ear) was automatically displayed, in Celsius degree, on the thermal image markers of the software, yielding two values for each study pig.

\section{Statistical Analysis}

Data were downloaded to Microsoft ${ }^{\circledR}$ Excel $^{\circledR}$ for Mac OS where descriptive statistics were calculated. All other statistical analyses were conducted using SAS 9.3 (SAS Institute, Inc., Cary, NC, USA). The Shapiro test was used on the model residual information as well as the examination of the normal plot to evaluate the normal distribution.

\section{Skin temperature of pigs with tail lesions}

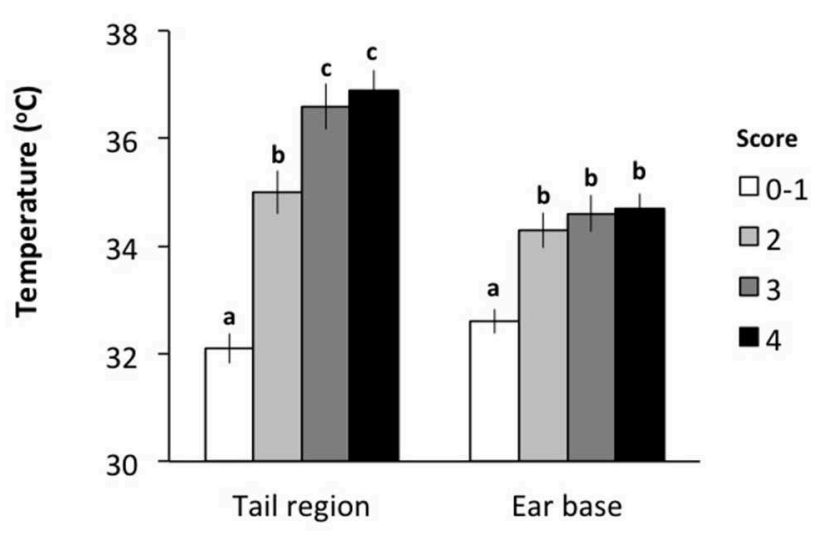

FIGURE 3 | Skin temperature at the base of the tail and the ear of pigs within different tail lesion severity measured in the lairage. Different letters $(a, b, c)$ represent significant differences $(P<0.05)$ between tail lesion scores.

The effect of tail lesion score and sex of the pig on the hotspot temperature of the selected infrared image areas, both at the base of the tail and the ear, were analyzed using linear mixed models (Proc Glimmix of SAS), using normal distribution. Tail lesion score and sex of the pig were included in the two models as fixed effects. For all models animal was considered as experimental unit. Interactions between tail lesion score and sex of the pig were removed from all models as they were not significant $(P$ $>0.10)$. Results are reported as least square means \pm standard error (S.E.) and statistic effects were reported when $P \leq 0.05$. Finally, Pearson's correlation coefficient was used to analyze the relationship between the hotspot temperature of the selected infrared image area of the base of the tail and the ear of the pigs.

\section{RESULTS}

There was an effect of the severity of tail lesion score on the skin temperature measured at the base of the tail and the ear 
(Figure 3). Skin temperature measured at the base of the tail was significantly lower for tails scored $0-1$ than for all other tail lesion scores $(P<0.05)$. Pigs with tail lesions scored 2 had significantly lower skin temperatures at the base of the tail than pigs with tail lesions scored 3 or $4(P<0.05)$ while there was no difference in skin temperature at the base of the tail between pigs with tail lesion scores 3 and $4(P>0.05)$. Skin temperature measured at the ear base was significantly lower for tails scored $0-1$ than for all other tail lesion scores $(P<$ $0.05)$ with no difference between the other scores $(P>0.05)$. Sex did not affect the skin temperature of pigs recorded at the base of the tail and the ear $(P>0.05)$. Furthermore, there was a positive association between both measurements $(r=0.50 ; P$ $<0.001$ ), such that increasing tail lesion severity was associated with increases in skin temperature measured at the base of the tail and the ear.

\section{DISCUSSION}

The aim of the current study was to evaluate potential differences in skin temperature of tail bitten pigs compared to pigs with intact tails measured at the base of the tail and of the ear by infrared thermography camera. The study also evaluated the association between temperatures recorded at the two locations. The present study confirms the hypothesis that increasing severity of tail damage is associated with an increase in skin temperature at both regions. As far as we know, this is the first study to investigate the skin temperature of pigs with tail lesions of differing severity.

Less severe tail injuries caused by tail biting are more visible on the carcass than in live animals (39-42). Indeed Carroll et al. (42) showed that tail lesions of every severity category became more visible after scalding and dehairing due to the removal of dirt and hair. However, we were interested in associations with body temperature and hence had to work with live animals. While the normal body temperature range in resting pigs varies between 37.0 and $39.6^{\circ} \mathrm{C}(30)$ and the normal rectal temperature in the thermoneutral zone is $39.3^{\circ} \mathrm{C}(34)$, the temperature of the inside surface of the pig ear ranges from 27.3 to $35.0^{\circ} \mathrm{C}$, with a mean of $31.0 \pm 1.32^{\circ} \mathrm{C}$ (33). In pigs, temperature at the ear base is considered to give the best correlation between skin and rectal temperatures (34). Measured by a needle probe inserted under the skin, Soerensen et al. (38) reported $34.8^{\circ} \mathrm{C}$ as the highest temperature measured on the ear base of a living sow. In the current study, the average skin temperature at the ear base was $34.3 \pm 0.33$ (score 2), $34.6 \pm 0.34$ (score 3 ) and $34.7 \pm 0.28^{\circ} \mathrm{C}$ (score 4 ). These are similar to the values reported by Soerensen et al. (38) in healthy sows under experimental conditions. We expected to find higher skin temperatures in our study pigs due to the severity of the tail lesions; but it is important to highlight that measurements were taken after transportation from the farm and that the pigs were lairaged in pens with open walls such that the pigs were exposed to the weather. Unfortunately, the ambient temperature and humidity parameters in the lairage were not recorded in each experimental day, but the thermography camera automatically adjusted these data when measuring the surface temperature. On the other hand, to minimize the effect of the experimental day and the ambient temperature on the skin temperature of the pigs, pigs with the five different tail lesion scores were scored and imaged in all experimental days.

In the present study, there was an association between tail lesion severity and the skin temperature, such that increasing tail lesion score was associated with an increase in skin temperature at both the tail and ear base. This association is in agreement with Heinonen et al. (8) who found that the systemic inflammatory response seemed to be more severe in animals with deep tail inflammation. Tail biting induces a histopathologically detectable inflammation of the tail-end in slaughter pigs and leads to the spread of infection (8). Therefore, in the present study, it is suggested that the higher values of skin temperature at the tail base indicate signs of inflammation and potential infection (8), explaining the association between severity of tail lesion and the skin temperature at the base of the tail.

Previous studies showed that infection from the tail area to the sacral vertebrae via the lymph or blood stream causes pyemic foci in other parts of the body, especially lungs and vertebrae (43). Tail bitten pigs show higher serum protein concentrations and a higher prevalence of anemia than uninjured pigs (6), indicating that victims of tail biting are more severely challenged by (bacterial) infections (44) and chronic inflammation (45), finally showing the elevated systemic temperatures. Therefore, tail wounds from biting appear to cause infection elsewhere in the body (22). Unfortunately we did not measure either local or general infection and, therefore, such effect warrants further research. Furthermore, despite our study did not include physiological parameters, Warris et al. (33) also reported a positive correlation between the temperature of the inside surface of the ear and the activity of serum creatine kinase, as well as between concentrations of serum cortisol and blood temperature, suggesting that the hotter pigs were suffering from higher levels of stress. Stress $(46,47)$ and inflammation $(48)$ cause an increase in body temperature. Stress (49) and fever (36) significantly elevate the skin temperature in pigs, although with a delay compared to rectal temperature. Schmidt et al. (50) were able to detect febrile sows from eye and ear base temperatures using infrared thermography images. Increases in skin temperature were also reported in pigs with palpable symptoms of osteoarthrosis (51) and with high blood temperature at exsanguination (33).

From the current study, it is important to highlight that pigs with moderate tail lesions (score 2) showed a significantly higher skin temperature compared to pigs with intact tails or mild tail lesions. This finding suggests that even pigs with moderate tail lesions (score 2) may have general inflammation and infection. This is in agreement with Smith and Penny (52) who suggest that even tail damage restricted to puncture wounds can readily set up pyemia. The Scientific Report of EFSA (4) highlighted the importance of recording both moderate and severe tail damage at meat inspection. Previous studies showed that pigs with even moderate tail lesions have higher odds of having abscesses or pleurisy/embolic pneumonia (21), pathological 
lesions, carcass trimmings/condemnations and reduced carcass weight $(12,20,22,24)$. Moreover, even for carcasses without any disease potentially detected at post-mortem inspection, Teixeira et al. (22) reported a reduction in carcass weight associated with tail lesions scored as moderate or severe (scores $>2$ ).

Finally, the findings from the present study support that the infrared thermography camera may be used as a Precision Livestock Farming (PLF) tool for recognizing/detecting tail lesions of different severity in live pigs, with high value to the farmer in managing tail biting on farm, especially in undocked pigs.

\section{CONCLUSION}

This study showed that the severity of tail lesions increases the skin temperature of pigs measured at the base of the tail or the ear. The findings suggest that even pigs with moderate tail lesions (score 2) may have general inflammation and infection, evidenced by the elevated systemic temperature compared to pigs with none or mild tail lesion (score $0-1$ ), which provides further support for the importance of more detailed measurements of tail lesion severity in pigs.

\section{DATA AVAILABILITY STATEMENT}

The datasets generated for this study are available on request to the corresponding author.

\section{REFERENCES}

1. Taylor NR, Main DCJ, Mendl M, Edwards SA. Tail-biting: a new perspective. Vet J. (2010) 186:137-47. doi: 10.1016/j.tvjl.2009.08.028

2. Bracke M, De Lauwere CC, Wind SM, Zonerland JJ. Attitudes of Dutch pig farmers towards tail biting and tail docking. J Agricul Environ Ethics. (2013) 26:847-68. doi: 10.1007/s10806-012-9410-2

3. Straw B, Zimmerman J, D'Allaire S, Taylor D. Diseases of Swine. Oxford: Blackwell Publishing Ltd (2006).

4. EFSA. The risks associated with tail biting in pigs and possible means to reduce the need for tail docking considering the different housing and husbandry systems-Scientific Opinion of the Panel on Animal Health and Welfare. EFSA J. (2007) 611:1-13. doi: 10.2903/j.efsa.2007.611

5. Smulders D, Verbeke G, Mormède P, Geers R. Validation of a behavioral observation tool to assess pig welfare. Physiol Behav. (2006) 89:438-47. doi: 10.1016/j.physbeh.2006.07.002

6. Munsterhjelm C, Simola O, Keeling L, Valros A, Heinonen M. Health parameters in tail biters and bitten pigs in a case-control study. Animal. (2013) 7:814-21. doi: 10.1017/S1751731112002194

7. Valros A, Munsterhjelm C, Puolanne E, Ruusunen M, Heinonen M, Peltoniemi OA, et al. Physiological indicators of stress and meat and carcass characteristics in tail bitten slaughter pigs. Acta Vet Scand. (2013) 55:75. doi: 10.1186/1751-0147-55-75

8. Heinonen M, Orro T, Kokkonen T, Munsterhjelm C, Peltoniemi O, Valros A. Tail biting induces a strong acute phase response and tail-end inflammation in finishing pigs. Vet J. (2010) 184:303-7. doi: 10.1016/j.tvjl.2009.02.021

9. Schrøder-Petersen DL, Simonsen HB. Tail-biting in pigs. Vet J. (2001) 162:196-210. doi: 10.1053/tvjl.2001.0605

10. Zonderland JJ, Bracke MBM, den Hartog LA, Kemp B, Spoolder HAM. Gender effects on tail damage development in single- or mixed-sex groups

\section{ETHICS STATEMENT}

This study was part of a research project approved by the Scientific Ethics Committee for Animals and Environmental Care of the Pontificia Universidad Católica de Chile.

\section{AUTHOR CONTRIBUTIONS}

$\mathrm{DT}, \mathrm{LB}$, and DE-H contributed to the concept of the work and interpreted data. DT and DE-H initiated, performed statistical analysis, and designed the study. DT performed the experiment and wrote the manuscript. LB and DE-H contributed to the manuscript. All authors approved the final version of the manuscript.

\section{FUNDING}

This study was funded by the National Commission for Scientific and Technological Research, CONICYT, through the Programme FONDECYT, grant number 11170822.

\section{ACKNOWLEDGMENTS}

The first author acknowledge the financial support provided by CONICYT, project number FONDECYT 11170822. Great thanks to all staff of the abattoir who always supported the development of the project. Many thanks to Patricia Camila Carrasco Gonzalez for assisting in conducting the experiment and for their help with data collection.

of weaned piglets. Livest Sci. (2010) 129:151-8. doi: 10.1016/j.livsci.201 0.01 .018

11. Sinisalo A, Niemi JK, Heinonen M, Valros A. Tail biting and production performance in fattening pigs. Livestock Sci. (2012) 143:220-5. doi: 10.1016/j.livsci.2011.09.019

12. Harley S, Boyle L, O'Connell N, More S, Teixeira D, Hanlon A. Docking the value of pigmeat? Prevalence and financial implications of welfare lesions in Irish slaughter pigs. Anim Welfare. (2014) 23:275-85. doi: 10.7120/09627286.23.3.275

13. Wallgren $\mathrm{P}$, Lindahl $\mathrm{E}$. The influence of tail biting on performance of fattening pigs. Acta Vet Scand. (1996) 37:453-60.

14. Moinard C, Mendl M, Nicol CJ, Green LE. A case control study of on-farm risk factors for tail biting in pigs. Appl Anim Behav Sci. (2003) 81:333-55. doi: 10.1016/S0168-1591(02)00276-9

15. Olsson I, De Jonge F, Schuurman T, Helmond F. Poor rearing conditions and social stress in pigs: repeated social challenge and the effect on behavioural and physiological responses to stressors. Behav Processes. (1999) 46:201-15. doi: 10.1016/S0376-6357(99)00036-4

16. Dalmau A, Temple D, Rodríguez P, Llonch P, Velarde A. Application of the Welfare Quality® protocol at pig slaughterhouses. Anim Welfare. (2009) 18:497-505.

17. Sutherland MA, Tucker CB. The long and short of it: A review of tail docking in farm animals. Appl Anim Behav Sci. (2011) 135:179-91. doi: 10.1016/j.applanim.2011.10.015

18. Huey R. Incidence, location and interrelationships between the sites of abscesses recorded in pigs at a bacon factory in Northern Ireland. Vet Rec. (1996) 138:511-4. doi: 10.1136/vr.138. 21.511

19. Elbers ARW, Tielen MJM, Snijders JMA, Cromwijk WAJ, Hunneman WA. Epidemiological studies on lesions in finishing pigs in the Netherlands. I 
prevalence, seasonality and interrelationship. Prevent Vet Med. (1992) 14:21731. doi: 10.1016/0167-5877(92)90018-B

20. Kritas SK, Morrison RB. Relationships between tail-biting in pigs and disease lesions and condemnations at slaughter. Vet Rec. (2007) 160:149-52. doi: $10.1136 /$ vr.160.5.149

21. Marques BMF, Bernardi ML, Coelho CF, Almeida M, Morales OE, Mores TJ, et al. Influence of tail biting on weight gain, lesions and condemnations at slaughter of finishing pigs. Pesq Vet Brasil. (2012) 32:96774. doi: $10.1590 / \mathrm{S} 0100-736 \mathrm{X} 2012001000003$

22. Teixeira DL, Harley S, Hanlon A, O'Connell NE, More S, Garcia Manzanilla E, et al. Study on the association between tail lesion score, cold carcass weight and viscera condemnations in slaughter pigs. Front Vet Sci. (2016) 3:24. doi: $10.3389 /$ fvets.2016.00024

23. Valros A, Ahlström S, Rintala $H$, Häkkinen $T$, Saloniemi $H$. The prevalence of tail damage in slaughter pigs in Finland and associations to carcass condemnations. Acta Agricul Scand. (2004) 54:213-9. doi: 10.1080/09064700510009234

24. van Staaveren N, Vale AP, Manzanilla EG, Teixeira DL, Leonard FC, Hanlon A, et al. Relationship between tail lesions and lung health in slaughter pigs. Prev Vet Med. (2016) 127:21-6. doi: 10.1016/j.prevetmed.2016.03.004

25. Swaby H, Gregory NG. A note on the frequency of gastric ulcers detected during post-mortem examination at a pig abattoir. Meat Sci. (2012) 90:269-71. doi: $10.1016 /$ j.meatsci.2011.06.015

26. Reimert I, Rodenburg TB, Ursinus WW, Kemp B, Bolhuis JE. Selection based on indirect genetic effects for growth, environmental enrichment and coping style affect the immune status of pigs. PLoS ONE. (2014) 9:e108700. doi: 10.1371/journal.pone. 0108700

27. Proudfoot K, Habing G. Social stress as a cause of diseases in farm animals: Current knowledge and future directions. Vet J. (2015) 206:15-21. doi: 10.1016/j.tvjl.2015.05.024

28. Van den Berg A, Brülisauer F, Regula G. [Prevalence of gastric lesions in the pars proventricularis in finishing pigs at slaughter in Switzerland]. Schweiz Arch Tierheilkd. (2005) 147:297-303. doi: 10.1024/0036-7281.147.7.297

29. Siewert C, Dänicke S, Kersten S, Brosig B, Rohweder D, Beyerbach M, et al. Difference method for analysing infrared images in pigs with elevated body temperatures. Zeitschrift für Medizinische Physik. (2014) 24:6-15. doi: 10.1016/j.zemedi.2013.11.001

30. Hannon JP, Bossone C, Wade C. Normal physiological values for conscious pigs used in biomedical research. Lab Anim Sci. (1990) 40:293-8.

31. Gariepy C, Amiot J, Nadai S. Ante-mortem detection of PSE and DFD by infrared thermography of pigs before stunning. Meat Sci. (1989) 25:37-41. doi: 10.1016/0309-1740(89)90064-8

32. Knowles TG, Brown SN, Edwards JE, Warriss PD. Ambient temperature below which pigs should not be continuously showered in lairage. Vet Rec. (1998) 143:575-8. doi: 10.1136/vr.143.21.575

33. Warriss P, Pope S, Brown S, Wilkins L, Knowles T. Estimating the body temperature of groups of pigs by thermal imaging. Vet Rec. (2006) 158:331-4. doi: 10.1136/vr.158.10.331

34. Soerensen DD, Pedersen LJ. Infrared skin temperature measurements for monitoring health in pigs: a review. Acta Vet Scand. (2015) 57:5. doi: 10.1186/s13028-015-0094-2

35. McManus C, Tanure CB, Peripolli V, Seixas L, Fischer V, Gabbi AM, et al. Infrared thermography in animal production: an overview. Comp Electron Agricul. (2016) 123:10-6. doi: 10.1016/j.compag.2016.01.027

36. Loughmiller JA, Spire MF, Dritz SS, Fenwick BW, Hosni MH, Hogge SB. Relationship between mean body surface temperature measured by use of infrared thermography and ambient temperature in clinically normal pigs and pigs inoculated with Actinobacillus pleuropneumoniae. Am J Vet Res. (2001) 62:676-81. doi: 10.2460/ajvr.2001.62.676

37. Harley S, More S, O'Connell N, Hanlon A, Teixeira D, Boyle L. Evaluating the prevalence of tail biting and carcase condemnations in slaughter pigs in the Republic and Northern Ireland, and the potential of abattoir meat inspection as a welfare surveillance tool. Vet Rec. (2012) 171:621-621. doi: $10.1136 /$ vr.100986
38. Soerensen DD, Clausen S, Mercer JB, Pedersen LJ. Determining the emissivity of pig skin for accurate infrared thermography. Comp Electr Agricul. (2014) 109:52-8. doi: 10.1016/j.compag.2014.09.003

39. Velarde A, Dalmau A, Fàbrega E, Manteca X. Health and welfare management of pigs based on slaughterline records. In: The 56th Annual Meeting of the European Association for Animal Production. Uppsala (2005). p. 2-5.

40. Harley S, More S, Boyle L, O'Connell N, Hanlon A. Good animal welfare makes economic sense: potential of pig abattoir meat inspection as a welfare surveillance tool. Ir Vet J. (2012) 65:1-12. doi: 10.1186/2046-0481-65-11

41. van Staaveren N, Teixeira DL, Hanlon A, Boyle LA. The effect of mixing entire male pigs prior to transport to slaughter on behaviour, welfare and carcass lesions. PLoS ONE. (2015) 10:e0122841. doi: 10.1371/journal.pone.0122841

42. Carroll G, Boyle L, Teixeira D, Van Staaveren N, Hanlon A, O'Connell N. Effects of scalding and dehairing of pig carcasses at abattoirs on the visibility of welfare-related lesions. Animal. (2016) 10:460-7. doi: 10.1017/S1751731115002037

43. Norval J. Abscesses in pigs. Vet Rec. (1966) 78:708-13. doi: 10.1136/vr.78.21.708

44. Odink J, Smeets J, Visser I, Sandman H, Snijders J. Hematological and clinicochemical profiles of healthy swine and swine with inflammatory processes. J Anim Sci. (1990) 68:163-70. doi: 10.2527/1990.681163x

45. Friendship RM, Lumsden J, McMillan I, Wilson M. Hematology and biochemistry reference values for Ontario swine. Can J Comp Med. (1984) 48:390.

46. Broom D. Welfare assessment and welfare problem areas during handling and transport. Livestock Handling Transport. (2000) 2:43-61. doi: 10.1079/9780851994093.0043

47. Schäfer A, Rosenvold K, Purslow PP, Andersen HJ, Henckel P. Physiological and structural events post mortem of importance for drip loss in pork. Meat Sci. (2002) 61:355-66. doi: 10.1016/S0309-1740(01)00205-4

48. Tesch T, Bannert E, Kluess J, Frahm J, Hüther L, Kersten S, et al. Relationships between body temperatures and inflammation indicators under physiological and pathophysiological conditions in pigs exposed to systemic lipopolysaccharide and dietary deoxynivalenol. J Anim Physiol Anim Nutr. (2018) 102:241-51. doi: 10.1111/jpn.12684

49. Magnani D, Gatto M, Cafazzo S, Stelletta C, Morgante M, Costa L. Difference of surface body temperature in piglets due to the backtest and environmental condition. In: Animal Hygiene and Sustainable Livestock Production. Proceedings of the XVth International Congress of the International Society for Animal Hygiene. Vienna, Austria: Tribun EU. (2011). p. 1029-1032.

50. Schmidt M, Lahrmann K-H, Ammon C, Berg W, Schön P, Hoffmann G. Assessment of body temperature in sows by two infrared thermography methods at various body surface locations. I Swine Health Product. (2013) 21:203-9.

51. Sabec D, Lazar P. Preliminary results of noncontact temperature measuring using an infrared thermometer on the tarsus pf swine with osteoarthrosis tarsi deformans. DTW Dtsch Tierarztl Wochenschr. (1990) 97:43-4.

52. Smith W, Penny R. Tail-biting and tail-docking in pigs. Vet Rec. (1998) 142:496.

Conflict of Interest: LB was employed by the company Pig Development Department, Moorepark, TEAGASC.

The remaining authors declare that the research was conducted in the absence of any commercial or financial relationships that could be construed as a potential conflict of interest.

Copyright $\odot 2020$ Teixeira, Boyle and Enríquez-Hidalgo. This is an open-access article distributed under the terms of the Creative Commons Attribution License (CC $B Y)$. The use, distribution or reproduction in other forums is permitted, provided the original author(s) and the copyright owner(s) are credited and that the original publication in this journal is cited, in accordance with accepted academic practice. No use, distribution or reproduction is permitted which does not comply with these terms. 